\title{
Unsteady Aerodynamic Modeling in Roll for the NASA Generic Transport Model
}

\author{
Patrick C. Murphy* \\ NASA Langley Research Center, Hampton, VA, 23681-2199 \\ Vladislav Klein ${ }^{\dagger}$ \\ National Institute of Aerospace, NASA Langley Research Center, Hampton, VA, 23681-2199 \\ Neal T. Frink \\ NASA Langley Research Center, Hampton, VA, 23681-2199
}

\begin{abstract}
Reducing the impact of loss-of-control conditions on commercial transport aircraft is a primary goal of the NASA Aviation Safety Program. One aspect in developing the supporting technologies is to improve the aerodynamic models that represent these adverse conditions. Aerodynamic models appropriate for loss of control conditions require a more general mathematical representation to predict nonlinear unsteady behaviors. In this paper, a more general mathematical model is proposed for the subscale NASA Generic Transport Model (GTM) that covers both low and high angles of attack. Particular attention is devoted to the stall region where full-scale transports have demonstrated a tendency for roll instability. The complete aerodynamic model was estimated from dynamic wind-tunnel data. Advanced computational methods are used to improve understanding and visualize the flow physics within the region where roll instability is a factor.
\end{abstract}

\section{Nomenclature}

$\begin{array}{llll}\mathrm{A}_{\mathrm{j}}, \mathrm{B}_{\mathrm{j}} & =\text { Fourier coefficients } & \mathrm{T} & =\text { dimensional time constant, sec } \\ a, \mathrm{~b}_{1} & =\text { deficiency function parameters } & t & =\text { time, sec } \\ b & =\text { wing span, ft } & V & =\text { velocity, fps } \\ C_{l} & =\text { rolling-moment } & \alpha & =\text { angle of attack, rad or deg } \\ C_{n} & =\text { yawing-moment coefficient } & \alpha_{0} & =\text { initial angle of attack in forced- } \\ C_{Y} & =\text { side-force coefficient } & & \text { oscillation experiments, rad or deg } \\ \bar{c} & =\text { mean aerodynamic chord, ft } & \beta & =\text { sideslip angle, rad or deg } \\ F_{a \beta} & =\text { deficiency functions } & \eta & =\text { state variable } \\ f & =\text { frequency, Hz } & \sigma & =\text { standard error } \\ k & =\text { reduced frequency, } \pi b f / V & \tau & =\text { dummy integration variable } \\ m & =\text { no. of harmonics in Fourier expansion } & & \\ N & =\text { number of data points } & & =\text { non-dimensional time constant, } \frac{1}{b_{1}}\left(\frac{2 V}{b}\right) \\ p, r & =\text { roll and yaw rates, rad/sec } & \phi & =\text { roll angle, rad or deg } \\ R^{2} & =\text { coefficient of determination } & \psi & =\text { yaw angle, rad or deg } \\ S & =\text { reference area, ft } & & \\ \mathrm{s} & =\text { estimated standard error } & \omega & =\text { angular frequency, rad } / \mathrm{sec}\end{array}$

\footnotetext{
* Senior Research Engineer, Dynamic Systems \& Control Branch, MS 308, Associate Fellow.

${ }^{\dagger}$ Professor Emeritus, Dynamic Systems \& Control Branch, MS 308, Associate Fellow.

* Senior Research Engineer, Configuration Aerodynamics Branch, MS 499, Associate Fellow.
} 


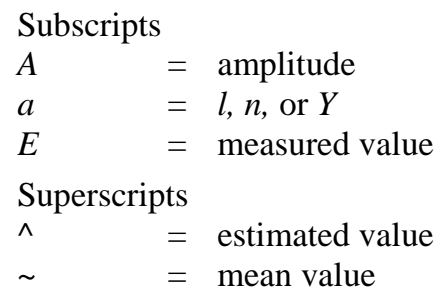

Aerodynamic Derivatives

$$
\begin{aligned}
& C_{a_{\beta}}=\frac{\partial C_{a}}{\partial \beta} \text { where } a=l, n, \text { or } Y \\
& C_{a_{p}}=\frac{b}{2 V} \frac{\partial C_{a}}{\partial p}, C_{a_{r}}=\frac{b}{2 V} \frac{\partial C_{a}}{\partial r}
\end{aligned}
$$

\section{Introduction}

$\mathrm{R}$ educing the impact of loss-of-control conditions on commercial transport aircraft is a primary goal of the NASA Aviation Safety Program. In support of this goal, both analytical and experimental technologies continue to be developed to improve the aerodynamic models that represent these adverse conditions. Aerodynamic models appropriate for loss-of-control conditions require a more general mathematical representation to predict nonlinear unsteady behaviors. Researchers are addressing this problem by developing analytical tools in Computational Fluid Dynamics (CFD) and experimental tools using direct measurement of aircraft responses in flight and in wind tunnels along with System Identification (SID) technology to determine mathematical models.

A number of experimental and analytical studies have been performed to obtain aerodynamic models for the subscale NASA Generic Transport Model (GTM) and this work has produced significant improvements in simulating transport aircraft responses in loss-of-control conditions. Early studies to improve GTM simulation in upset conditions were reported by Cunningham et al. ${ }^{1}$ in 2004 and Foster et al. ${ }^{2}$ in 2005. In these studies an expanded aerodynamic data base was produced and both static and dynamic roll-axis instabilities and nonlinearities were identified at stall angles of attack. Later in 2010, Belcastro and Foster ${ }^{1}$ provided a study showing that loss of control is still the leading cause of accidents worldwide. In 2011, Advani and Field ${ }^{4}$ highlighted the need for integrated upset prevention and recovery training in flight simulators.

With this motivation, both computational and experimental research in modeling transport aircraft has continued. A recent paper by Morelli in 2012 produced global aerodynamic models for GTM using flight test data. The results were obtained using automated perturbation inputs and were presented over a large range of angle of attack. Instability was found and characterized as a roll-off departure at $14^{\circ}$ angle of attack. These results are consistent with the modeling work by Murphy and Klein ${ }^{6}$ where dynamic roll instability was found near $12^{\circ}$ angle of attack. In Ref 6 results were obtained using advanced modeling methods applied to dynamic forced-oscillation tests of the GTM. In this later study, nonlinear unsteady responses were identified in the stall regime. The strength of this roll instability and the abrupt nature of the roll-off departure warranted further investigation.

In this study both CFD and SID approaches were used to investigate the subscale NASA GTM roll-axis aerodynamics. The aerodynamic model was estimated from dynamic wind-tunnel data and additional insights into the flow physics were obtained using advanced computational methods where roll instability and unsteady behaviors are a factor. The SID approach is discussed by presenting a simplified version of a more general formulation of the transport aerodynamic model structure using indicial models ${ }^{7}$. The identification problem is pursued by first performing harmonic analysis to locate and assess the severity of nonlinear and unsteady behaviors. This analysis helped identify appropriate model structure for parameter estimation. Although the global model for rolling moment is nonlinear, local models identified by dynamic tests must be evaluated to determine their adequacy as linear or nonlinear representations over the range tested. In this study only local models are developed with appropriate model structures to reflect the degree of nonlinearity and unsteadiness. These models, however, can form a foundation for a global model in simulation. Final parameter estimation was accomplished by using output-error, as described in Ref. 8. Data filtering and its impact on harmonic analysis are discussed in detail to clarify key modeling steps that lead to determination of linear or nonlinear model structures. Identifying unsteady aerodynamic models can be performed efficiently with advanced testing methods that use wide-band inputs ${ }^{9,10}$; however, for this study only conventional single-axis, single-frequency, forced-oscillation data are available from previous wind-tunnel studies.

Advances in computational methods offer an opportunity to complement the wind-tunnel data with physicsbased analyses that effectively provide an "analytical" wind tunnel. These methods allow the use of advanced test techniques and motions not readily available when applying conventional experimental methods and thus allow more flexibility in understanding and estimating key aerodynamic modeling parameters. The combined experimental and analytical experiments offer an opportunity to gain greater insights into aerodynamic phenomena observed in flight. An initial investigation demonstrating the potential benefits of this approach was reported in Ref. 11, where 
linear unsteady aerodynamic models were identified from both wind-tunnel measurements and CFD simulations. Aerodynamic model comparisons were obtained using the NATO RTO AVT-161 SACCON ${ }^{12}$ wind-tunnel model.

\section{Wind Tunnel Measured Data}

Wind-tunnel tests were conducted at the NASA Langley 14x22 Wind Tunnel in 2009 to obtain lateral-directional data appropriate for identification of unsteady aerodynamic models. Analysis of this data was reported in Ref. 6, where analysis of the yaw-axis measurements was the primary focus. A $5.5 \%$ scale model, representing a conventional twin-engine commercial transport, was tested. Figure 1 shows the basic geometry of the model. Roll and yaw forced oscillation experiments were performed over a wide range of frequencies and amplitudes to facilitate unsteady model identification. For the roll case, the tunnel speed was $92 \mathrm{fps}$ and measurements were made for roll oscillations at angles of attack from -5 to 75 degrees; five frequencies of 0.06, 0.12, 0.23, 0.46, and $0.92 \mathrm{~Hz}$; and four amplitudes of 5, 10,20, and 30 degrees. The frequencies chosen correspond to the non-dimensional

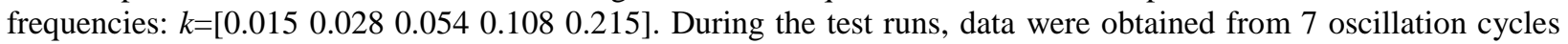
for low frequency data and up to 44 cycles for the remaining data. The sample rate was $250 \mathrm{~Hz}$ with a low-pass 100 $\mathrm{Hz}$ analog filter. The resulting data were further filtered with a $4 \mathrm{~Hz}$ low-pass digital filter to remove unwanted frequency content. The $4 \mathrm{~Hz}$ filter was run in both directions to ensure no phase error was added to the data.

\section{CFD Simulated Data}

CFD predictions were performed with the USM3D flow solver ${ }^{13}$ that is part of the NASA Tetrahedral Unstructured Software System (TetrUSS) ${ }^{14}$. USM3D is a parallelized tetrahedral cell-centered, finite volume compressible Reynolds-averaged Navier-Stokes (RANS) flow solver. The term "cell centered" means that the finite volume flow solution is solved at the centroid of each tetrahedral cell. Inviscid flux quantities are computed across each tetrahedral cell face using various upwind schemes. Spatial discretization is accomplished by a novel reconstruction process, based on an analytical formulation for computing solution gradients within tetrahedral cells. The solution can be advanced in time by a second order "physical" time step scheme, a second order "dual" time step scheme, or to a steady-state condition by an implicit backward-Euler scheme. Several turbulence models are available including the one-equation Spalart-Allmaras (SA) model and several two-equation models. The twoequation models available are the Jones and Launder $k-\varepsilon$ model, Menter Shear Stress Transport (SST) model, nonlinear Algebraic Reynolds Stress Models (ARSM) of Girimaji and Shih/Zhu/Lumley, and the Wilcox $1988 k-\omega$ model. Detached Eddy Simulation (DES) has been implemented in all of the turbulence models. A capability to trip the flow at specified locations on aerodynamic surfaces has been implemented for the $k-\varepsilon$ turbulence model, but fully turbulent flow was assumed for the results in this paper. USM3D has capabilities for overset grids and dynamic grid motion, the latter being used in the current study. Published guidelines ${ }^{15}$ for computing dynamic forced oscillation solutions were employed for the CFD computations in this study. CFD simulations were generated that correspond to the roll forced-oscillation wind-tunnel tests performed on the GTM in the regions of interest.

\section{Aerodynamic Modeling}

Simplified model equations for the lateral coefficients $C_{l}, C_{n}$, and $C_{Y}$ represented by $C_{a}$, where $a=l, n$, or $Y$,

can be developed from a general form of the indicial model equations ${ }^{7,16-18}$. Each coefficient is considered in the form

$$
C_{a}(t)=C_{a}(0)+\int_{0}^{t} C_{a_{\beta}}(t-\tau) \dot{\beta}(\tau) d \tau+\frac{b}{2 V} \int_{0}^{t} C_{a_{p}}(t-\tau) \dot{p}(\tau) d \tau+\frac{b}{2 V} \int_{0}^{t} C_{a_{r}}(t-\tau) \dot{r}(\tau) d \tau
$$

where $C_{a_{\beta}}(t), C_{a_{p}}(t)$, and $C_{a_{r}}(t)$ are the indicial functions and $C_{a}(0)$ is the initial value of $C_{a}$. Two assumptions were adopted to simplify the model: the effect of angular accelerations $\dot{p}$ and $\dot{r}$ on any coefficient can be neglected and the indicial functions in Eq. (1) can be expressed as

$$
C_{a_{\beta}}(t)=C_{a_{\beta}}(\infty)-F_{a_{\beta}}(t)
$$


where $F_{a_{\beta}}(t)$ is the deficiency function and $C_{a_{\beta}}(\infty)$ is the partial derivative of $C_{a}$ with respect to $\beta$ evaluated in steady flow conditions.

The simplified model, which takes into account changes with respect to steady state, has the form

$$
C_{a}(t)=C_{a_{\beta}}(\infty) \beta(t)+\frac{b}{2 V} C_{a_{p}}(\infty) p(t)+\frac{b}{2 V} C_{a_{r}}(\infty) r(t)-\int_{0}^{t} F_{a_{\beta}}(t-\tau) \dot{\beta}(\tau) d \tau
$$

where $C_{a}(0)$ has been subtracted from both sides of Eq. (3). To obtain a model appropriate for identification and with a limited number of parameters, the deficiency function is assumed to be a simple exponential function ${ }^{16}$

$$
F_{a_{\beta}}=a e^{-b_{1} t}
$$

Models appropriate for an aircraft undergoing one degree of freedom forced oscillation in roll or yaw can be obtained using Eqs. (3) and (4). Considering one degree of freedom rolling motion in the tunnel

$$
C_{a}(t)=C_{a}[\phi(t), p(t)]
$$

where roll angle is related to the sideslip angle by the equation

$$
\beta(t)=\sin ^{-1}(\sin \alpha \sin \phi(t))
$$

Combining Eqs. (3-6), the aerodynamic models can be formulated as

$$
C_{a}(t)=C_{a_{\beta}}(\infty) \beta(t)+\frac{b}{2 V} C_{a_{p}}(\infty) p(t)-a \int_{0}^{t} e^{-b_{1}(t-\tau)} \dot{\beta}(\tau) d \tau
$$

By introducing

$$
\eta(t)=\int_{0}^{t} e^{-b_{1}(t-\tau)} \dot{\beta}(\tau) d \tau
$$

and applying the Leibnitz integral rule, the state space form of Eq. (7) can be written as

$$
\begin{gathered}
\dot{\eta}(t)=-b_{1} \eta(t)+\dot{\beta}(t) \\
C_{a}(t)=C_{a_{\beta}}(\infty) \beta(t)+\frac{b}{2 V} C_{a_{p}}(\infty) p(t)-a \eta(t) .
\end{gathered}
$$

From Eq. (7), a steady response can be obtained ${ }^{16}$ as

$$
C_{a}(t)=\bar{C}_{a_{\beta}} \phi_{A} \sin (\omega t)+\bar{C}_{a_{p}} \phi_{A} k \cos (\omega t)
$$

where $\phi_{A}$ is the amplitude of roll oscillation, $k$ is reduced frequency, and $\bar{C}_{a_{\beta}}$ and $\bar{C}_{a_{p}}$ are the in-phase and out-ofphase components, respectively. These components are related to the model parameters by the equations ${ }^{17}$

$$
\bar{C}_{a_{\beta}}=C_{a_{\beta}}(\infty) \sin \alpha_{0}-a \frac{\tau_{1}^{2} k^{2}}{1+\tau_{1}^{2} k^{2}} \sin \alpha_{0}
$$




$$
\bar{C}_{a_{p}}=C_{a_{p}}(\infty)-a \frac{\tau_{1}}{1+\tau_{1}^{2} k^{2}} \sin \alpha_{0} .
$$

where $\tau_{1}$ is the non-dimensional inverse of $b_{1}$.

\section{Results and Discussion}

Before identifying the unsteady models described in the last section, analysis is required to determine where, with respect to angle of attack and sideslip, the more general models are needed, if at all, and to determine if a nonlinear formulation is required. Harmonic analysis along with the coefficient of determination, $R^{2}$, is used for that step. Since $R^{2}$ is sensitive to noise, the analysis can be made more difficult with low signal/noise data. Before presenting model parameter estimates, some discussion is provided to help establish a best practice for harmonic analysis and examples are given to demonstrate the effects of noise. Non-zero ordinate values on all plots were removed in order to maintain proprietary agreements.

\section{A. Harmonic Analysis}

A method of harmonic analysis ${ }^{19}$ was applied to measured aerodynamic coefficients. A mathematical model for these coefficients is

$$
C_{a}(t)=A_{0}+\sum_{j=1}^{m} A_{j} \cos (j \omega t)+\sum_{j=1}^{m} B_{j} \sin (j \omega t) \quad a=l, n \text { or } \mathrm{Y}
$$

where $A_{0}, A_{j}$, and $B_{j}$ are the Fourier coefficients. The analysis provides estimates of these coefficients, their standard errors, and the coefficient of determination, $R^{2}$. For the model with linear aerodynamics and $A_{0}=0$, the aerodynamic in-phase and out-of-phase components can be expressed in terms of the coefficients $A_{1}$ and $B_{1}$. For the roll oscillation case the expressions are

$$
\begin{gathered}
\bar{C}_{a_{\beta}}=\frac{B_{1}}{\phi_{A}} \\
\bar{C}_{a_{p}}=\frac{A_{1}}{k \phi_{A}}
\end{gathered}
$$

where $\beta$ is related to $\phi$ by Eq. (6).

The coefficient of determination, $R^{2}$, indicates the fraction of variation in the measured data explained by the variation in the model and is defined as

$$
R^{2}=1-S S_{E} / \mathrm{SS}_{r}, \quad 0<R^{2}<1
$$

where

$$
S S_{E}=\sum_{i=1}^{N}\left[C_{a_{E}}(i)-\hat{C}_{a}(i)\right]^{2}
$$

is the residual sum of squares and

$$
S S_{r}=\sum_{i=1}^{N}\left[C_{a_{E}}(i)-\tilde{C}_{a}(i)\right]^{2}
$$


is the total sum of squares. $C_{a_{E}}(i), \hat{C}_{a}(i)$, and $\tilde{C}_{a}(i)$ are the measured, estimated, and mean values, respectively.

Results of harmonic analysis performed on roll oscillatory data with amplitudes of $10^{\circ}, 20^{\circ}$, and $30^{\circ}$ are presented in Fig. 2a, b, and c, respectively. These plots show in-phase and out-of-phase components against angle of attack at different frequencies and are plotted with the same scales. The plots indicate very limited frequency dependence (unsteady behavior) for the in-phase component for all three amplitudes. Frequency dependence in the damping term (out-of-phase component, $\bar{C}_{l_{p}}$ ) is present at $\alpha_{0}=12^{\circ}$ for all three amplitudes. For the small amplitude case frequency dependence is also present at all $\alpha_{0}$ above $24^{\circ}$. For the larger amplitudes the unsteady behavior splits into two regions: $24^{\circ} \leq \alpha_{0} \leq 30^{\circ}$ for amplitude $20^{\circ}$, and $26^{\circ} \leq \alpha_{0} \leq 30^{\circ}$ for amplitude $30^{\circ}$. Both larger amplitudes present some unsteady behavior for $\alpha_{0}>40^{\circ}$. Roll damping instabilities are present at $\alpha_{0}$ near $12^{\circ}, 14^{\circ}$, and above $30^{\circ}$ for the smaller amplitude and at $\alpha_{0}$ near $12^{\circ}, 14^{\circ}$, and above $40^{\circ}$ for the two larger amplitudes. As an example of yaw, and side force derivatives, similar analyses are shown in Fig. 3 for one amplitude, $\phi_{A}=20^{\circ}$. Unsteady behavior and instability occur in approximately the same $\alpha_{0}$ locations.

\section{B. $R^{2}$ Analysis}

As follows from Eqs. (17) to (19), the estimates of $R^{2}$ are influenced by the value and number of Fourier coefficients (harmonic order) in Eq. (14) and the measurement noise in $C_{a_{E}}(i)$. Because the Fourier coefficients are mutually orthogonal, the estimates of $A_{j}$ and $B_{j}$ will not change with the number of coefficients included in Eq. (14). Changes will only appear in the corresponding standard errors and residuals defined by Eq. (18). This makes $R^{2}$ an effective diagnostic tool to discern the adequacy of a linear first-order model against nonlinear higher harmonic models.

In Fig. 4a the harmonic analysis for the roll moment coefficient is repeated with $R^{2}$ shown for the middle amplitude case, $\phi_{A}=20^{\circ}$. Analysis was performed on data pre-filtered at $4 \mathrm{~Hz}$. At first look, $R^{2}$ indicates that substantial nonlinarity exists in the stalled flight regions for $12^{\circ} \leq \alpha_{0} \leq 40^{\circ}$. However, before making any observations on the details of this case, the effects of filtering should be discussed.

In Fig. $4 \mathrm{~b}$ the rolling moment coefficient in steady oscillation is presented as mean cycles of measured forcedoscillation data and a first order harmonic model. Before computing the mean cycle, three different band-pass filters were applied to produce data with substantially different noise levels. The corresponding harmonic models for each case are plotted (with same scales) to show the relationship of the data to its estimated linear model. Since a linear model can only produce a regular ellipse in this type of plot, ${ }^{17}$ any deviations from that regular shape indicate some degree of nonlinearity. The plots show that as filtering is done more aggressively, higher-order harmonics are removed. Consequently, engineering judgement is needed to avoid filtering too aggressively, removing information content and changing the resulting derivative estimates. In this example, the linear model provides a reasonable fit for even the least filtered $10 \mathrm{~Hz}$ data.

To further demonstrate the effect of filtering, the best use, and interpretation of $R^{2}$, Figs. 5 and 6 are presented. These figures show wind-tunnel measurement data filtered at $10 \mathrm{~Hz}$ and $2 \mathrm{~Hz}$ for side force and roll moment, respectively. In Fig. 5a, $R^{2}$ highlights poor adequacy of the linear model primarily for $10^{\circ} \leq \alpha_{0} \leq 30^{\circ}$, however, in this case filtering at $10 \mathrm{~Hz}$ has guaranteed the measurements were dominated by noise (or other responses outside the bandwidth of interest, such as structural vibrations) and in turn corrupted $R^{2}$. By filtering down to $2 \mathrm{~Hz}$, as shown in Fig. 5b with the same scales, a significant portion of the noise was removed and the $R^{2}$ metric now implies a linear model is completely adequate over the full range of $\alpha_{0}$. Only at $\alpha_{0}=0^{\circ}$ are $R^{2}$ values still low. This is caused by very small side force values at this condition resulting in very poor signal/noise levels. Also, in this case the aggressive filtering did not change the in-phase and out-of-phase coefficient values in any significant way.

The same treatment was performed on roll moment and the results are shown in Fig. 6. In Fig. 6a, with limited $10 \mathrm{~Hz}$ filtering, $R^{2}$ implies poor effectiveness of the linear model for $12^{\circ} \leq \alpha_{0} \leq 40^{\circ}$. In Fig. $6 \mathrm{~b}$, with $2 \mathrm{~Hz}$ filtering, the low $R^{2}$ range has been reduced to $14^{\circ} \leq \alpha_{0} \leq 35^{\circ}$ and the coefficients have not changed values compared with the $10 \mathrm{~Hz}$ filtering case. However, in the $2 \mathrm{~Hz}$ filtering case a number of conditions are still showing very low $R^{2}$ values. The three lowest $R^{2}$ cases at $\alpha_{0}=14^{\circ}, 22^{\circ}$, and $30^{\circ}$ are explained by considering measurement and harmonic model time histories plotted as response variable versus input variable. These three cases are representative of all the low $R^{2}$ cases so only these three will be discussed. The first case, at $\alpha_{0}=14^{\circ}$, demonstrates steady dynamics but with a nonlinear response; the second case, at $\alpha_{0}=22^{\circ}$, shows steady dynamics with a linear response; and the third case, at $\alpha_{0}=30^{\circ}$, presents unsteady dynamics with a linear response. For the set of amplitudes and frequencies presented in this study, the fourth possible case, a combination of nonlinear and unsteady responses, was not observed. An example of the fourth case was presented in Ref. 6, where a third order harmonic was required to 
model the cubic response in yawing moment to oscillations in sideslip. The source of the nonlinearity was found to be in the static term because of a strong cubic anti-symmetric relationship of yawing moment to sideslip. This type of model is common in the lateral-directional axis. In general, further investigation during the parameter estimation process is required to determine whether the nonlinearity is required in one or all three major model terms of Eq. (3). The three major terms are: (1) static terms, (2) steady-flow dynamic terms, and (3) unsteady terms.

Figure 7 shows rolling moment and side-force coefficients in steady oscillation for the three $\alpha_{0}$ cases with low $R^{2}$ at high and low frequencies and for both side force and roll moment. In order to highlight ellipse shapes the scales are not the same. The left side presents plots for the low frequency cases at $f=0.06 \mathrm{~Hz}$, and the right side shows high frequency cases at $f=0.92 \mathrm{~Hz}$. At the top of the figure are results at $\alpha_{0}=14^{\circ}$, where a low $R^{2}$ value occurred for the high frequency oscillations. In this case the low $R^{2}$ value correctly indicated a nonlinear response since the high frequency plot for $C_{l}$ is an irregular ellipse, and linear aerodynamics can only produce regular ellipses. Nonlinear responses are often expected in the lateral-directional axes where a strong cubic nonlinearity typically exists with respect to sideslip. This case is also a good example of where engineering judgement is required. Although the response at high frequency is nonlinear, respect for the very small values of the derivatives may lead to a conclusion that the added complexity of a nonlinear model is not warranted. $R^{2}$ does not account for overall magnitude of the response variables. The linear responses predicted by $R^{2}$ for $C_{Y}$ at both high and low frequencies are also confirmed.

For the second case at $\alpha_{0}=22^{\circ}$, the low $R^{2}$ value occurred at the lowest frequency. In this case noise has reduced $R^{2}$. Plots in the middle of Fig. 7 apply to this case and show that linear models match the measured data reasonably well except for the low frequency case of the roll moment, where the response measurement has substantial noise in spite of aggressive $2 \mathrm{~Hz}$ filtering. In this case, although the noise has reduced the $R^{2}$ metric, the estimated linear model is still adequate.

The third case at $\alpha_{0}=30^{\circ}$, presents a case where an unsteady model is required and a low $R^{2}$ value occurred at the lowest frequency (as shown in Fig. 6). Plots at the bottom of Fig. 7 apply to this case and the plots show linear models match the measured data reasonably well except for the low frequency case of the roll moment where the response measurement has substantial noise. Once again, although the noise has corrupted the $R^{2}$ metric, the estimated linear model is still adequate. Although the lag in response is not obvious, comparing the second and third cases, Fig. 6 highlights the unsteady model requirement due to frequency dependence for the third case at $\alpha_{0}=30^{\circ}$.

Figures 5, 6, and 7 highlight three cautions when using $R^{2}$ : (1) the metric $R^{2}$ is sensitive to signal-to-noise levels; (2) the metric $R^{2}$ does not account for the magnitude of response variables, so engineering judgement is required to evaluate if the response levels should be modeled; (3) model derivatives should not change values with appropriate filtering; consequently sensitivity of derivative estimates should be checked against the degree of digital filtering. Maintaining a factor of 5 between the digital filter cutoff frequency and the unsteady model pole locations has proven useful. Although filtering at $2 \mathrm{~Hz}$ did not change the in-phase and out-of-phase coefficients for this study, in practice $4 \mathrm{~Hz}$ has been found to be a good initial choice for filtering forced-oscillation data of rigid-body transport aircraft.

\section{CFD Simulation Analysis}

A previous study ${ }^{11}$ presented initial efforts to compare unsteady model parameters estimated using both CFD and dynamic wind-tunnel data. The promising results of that study have prompted the authors to extend the use of CFD simulation in this study. Although it is not the objective of this study to validate CFD, a number of additional insights into the physics behind the wind-tunnel results can be inferred, given the reasonably comparable aerodynamic predictions between CFD and wind-tunnel data. Further work is required to validate CFD applied to dynamic testing; however, with the use of CFD best practices ${ }^{15}$ researchers may obtain corroborating data or investigate conditions not readily possible with ground or flight test facilities. The solutions presented in the following were computed with the Reynolds Averaged Navier-Stokes equations using the Spalart-Allmaras turbulence model. The limitations of RANS for post-stall flows are well known. One objective of these computations is to assess the applicability of the RANS methodology in modeling the aggregate dynamic behavior of transport aircraft into and through stall. All solutions to follow, both static and dynamic, were advanced with second order physical time stepping.

Figure 8 provides a comparison of wind tunnel and CFD rolling-moment coefficient results for a range of angle of attack and frequency. These results correspond to GTM roll-forced sinusoidal oscillations with amplitude $20^{\circ}$. The oscillations were initiated from converged time-accurate static solutions at each angle of attack, and rotated for two complete oscillations. The dynamic solutions were essentially converged after one-fourth of a cycle. The $f=0.92$ $\mathrm{Hz}$ solution was advanced with 360 time steps per roll cycle, and the $f=0.12$ and $0.06 \mathrm{~Hz}$ cases with 1800 time 
steps per cycle. The red lines on Fig. 8 indicate the second roll cycle of the CFD simulation and black corresponds to the wind- tunnel mean-cycle measurements. Light gray lines show the wind-tunnel measurements before averaging the cycles of oscillation. These measurements highlight the substantial measurement noise and other responses outside the desired bandwidth at high angles of attack where separated flows dominate. Overall, a reasonable match in response shape is presented although at $\alpha_{0}=12^{\circ}$ some differences occur reflecting the sensitivity of modeling this difficult unsteady flight condition.

To further explain the modeling sensitivity near the unstable and unsteady condition at $\alpha_{0}=12^{\circ}$, Fig. 9 presents streamlines over the wing, that were computed to show how the flow is changing around this flight condition. These static solutions were advanced in time at a characteristic time step of 0.02 , i.e. a free stream particle will travel the length of the mean aerodynamic chord in 50 time steps. In addition to streamlines, lift and pitching moment static curves are presented below the wings along with the corresponding wind-tunnel data. Figure 9 shows a sequence of streamlines over the wing for four flight conditions: Fig. $9 \mathrm{a}, \alpha=4^{\circ}$, showing normal flight conditions; Fig. 9b, $\alpha=$ $11.75^{\circ}$, showing conditions just before a break in the lift curve; Fig. $9 \mathrm{c}, \alpha=12^{\circ}$, showing stalled conditions; and Fig. $9 \mathrm{~d}, \alpha=12.5^{\circ}$, showing post stall-break conditions. The figure shows that when the abrupt change in lift occurs at $\alpha=12^{\circ}$, streamlines also make a rapid change and indicate a stalled wing tip. Also in this region, the lift curve slope has become flat on either side of the break in lift. These adverse conditions support the unstable roll damping found in forced oscillation testing (Fig. 4) and is confirmed by abrupt roll departures demonstrated in GTM subscale flight tests near this angle of attack. CFD results point to a more abrupt change in lift and pitching moment than found in the wind-tunnel data.

CFD simulation was also used to generate more detailed forced-oscillation data over a limited range of $\alpha_{0}$ in key regions of interest. Two regions in $\alpha_{0}$ were computed that covered $10^{\circ} \leq \alpha_{0} \leq 15^{\circ}$ and $24^{\circ} \leq \alpha_{0} \leq 30^{\circ}$ with fine $1^{\circ}$ increments. Figure 10 presents harmonic analysis for CFD simulated roll forced-oscillation data. To reduce clutter, only high frequency results from the wind-tunnel analysis are overlaid for comparison. In general, the CFD analysis produced larger magnitudes for the coefficients but identified similar unsteady regions. The plots show that CFD results imply a shift of the largest roll instability toward $\alpha_{0}=14^{\circ}$ rather than at $\alpha_{0}=12^{\circ}$ as indicated by the windtunnel results. CFD results indicate unsteady behavior over a wider range of $11^{\circ} \leq \alpha_{0} \leq 15^{\circ}$, compared to the windtunnel result implying just at $\alpha_{0}=12^{\circ}$. In the lower $\alpha_{0}$ range $R^{2}$ analysis is similar to the wind-tunnel results. In that range both wind-tunnel and CFD capture the nonlinearity at high frequency. At the upper $\alpha_{0}$ range, the CFD results present higher values of $R^{2}$ reflecting the lower contribution of noise and other higher order effects in CFD simulation and confirming the use of linear unsteady models.

\section{Unsteady Model Parameter Estimation}

Parameter estimation was accomplished using a variation on an output-error $(\mathrm{OE})$ method $^{7,8,20}$ in the time domain. In the OE approach the model equations are given by Eqs. (9) and (10). Measured inputs, $\dot{\beta}$, and outputs, $C_{a}$, for each of the frequencies tested, are combined to ensure all the frequency content is included in the time domain estimation process. General application of the output-error method for aircraft is explained and relevant software is provided in Ref. 21.

Results from application of OE to GTM roll forced-oscillation data is shown in Fig. 11. This figure shows the estimated parameters and their $2 \sigma$ bounds for a large range of angle of attack, for $0^{\circ} \leq \alpha_{0} \leq 70^{\circ}$. Uncertainty bounds are generally very small and are in statistical agreement. This implies good information content or that adequate sensitivity was obtained for parameter estimation using the wind-tunnel data. The top plot shows steady-flow damping. Consistent with previous results, this term is neutral to unstable at $\alpha_{0}=12^{\circ}$ and $\alpha_{0} \geq 50^{\circ}$. The next two plots, in Fig. 11, define the unsteady term. The middle plot shows model parameter, $a$, that acts as a gain on the unsteady term and is, in general, relatively small except at $\alpha_{0}=12^{\circ}$. The bottom plot presents a non-dimensional time constant defining lag in the aerodynamic response. This plot shows three regions where unsteady behavior is present and the relative size of the lags in those regions. The smallest lag or fastest response is at $\alpha_{0}=12^{\circ}$.

As an example of model fidelity, at $\alpha_{0}=30^{\circ}$, response prediction is presented in Fig. 12. This example shows measured and unsteady model responses for both low and high frequency cases of the roll moment against sideslip. Response measurements plotted against $\phi$ are commonly used and have been presented in this study. However for this graphic, using $\beta$ highlights the adequacy of the linear unsteady model even with relatively large displacements of this key state variable. Equation (6) defines the relationship between $\phi$ and $\beta$. The estimated linear static curve is included to provide a reference line. Since linear models are used, the computed responses are regular ellipses. In general the models represent the measured data very well. One notable feature for the low frequency case is the very poor signal-to-noise for this test point. At the higher frequency, signal-to-noise is improved but still not very good. 
The measured data for the high frequency case does reflect some nonlinearity as indicated by the slightly irregular elliptical shape of the data; however, the linear model is still an adequate representation in this case. Although lag in the response caused by the unsteady flow is not readily visible in this type of plot, some evidence is presented by the rotation of the ellipse relative to the static line. For a linear model without a lag in the response, the long axis of the ellipse will align with the static line.

\section{Concluding Remarks}

This paper presents a study of roll-axis aerodynamics for the NASA GTM subscale vehicle. A method for harmonic analysis is presented and was performed on both experimental and computational force-oscillation data to identify where unsteady models are required and to characterize the linear or nonlinear nature of the models. Harmonic analysis was also applied to estimate cross derivatives and to determine the impact of amplitude effects. A best practice was suggested for harmonic analysis and $R^{2}$ analysis by appropriately filtering measurement data. A more detailed investigation of roll-axis instabilities was performed. The approach for investigating difficult aerodynamic flight regimes in aircraft suggested using both experimental and analytical technologies to provide greater insights into the aerodynamic phenomenon studied. Wind-tunnel dynamic data and simulated computational data showing reasonable comparisons support this approach. Computational methods provided aerodynamic details (dynamic force and moment data and streamlines) over a fine angle-of-attack grid near unsteady and unstable conditions. Computational data presented suggest the presence of roll instability (in agreement with the experimental analysis) and a lift curve stall break at $12^{\circ}$ angle of attack. The abrupt break in lift was not observed in the windtunnel results; however, flight testing does support the presence of an abrupt roll instability at this condition. Using the output-error estimation method, linear unsteady models were identified for the roll moment that can support a global nonlinear representation. An example of estimated model fidelity was demonstrated at a high angle of attack, $\alpha_{0}=30^{\circ}$, where unsteady aerodynamic behavior was found. The unsteady model represented the measured responses well.

Application of the unsteady modeling methodology to static and dynamic wind-tunnel data allows simulations of transport aircraft responses in adverse conditions to be developed and, in turn, facilitate development of control designs that provide stability and control in difficult flight regimes.

\section{Acknowledgments}

The authors extend their appreciation to the NASA Aviation Safety Program, Vehicle Systems Safety Project and the NASA Fundamental Aerodynamics Program, Subsonic Fixed Wing Project for support provided.

\section{References}

1. Cunningham, Kevin, Foster, John V, Shah, Gautam H., and Stewart, Eric C., "Simulation Study of a Commercial Transport Airplane During Stall and Post-Stall Flight," SAE Technical Paper Series 2004-01-3100, November 2004.

2. Foster, John V., Cunningham, Kevin, Fremaux, Charles M., Shah, Gautam H., and Stewart, Eric C., "Dynamics Modeling and Simulation of Large Transport Airplanes in Upset Conditions," AIAA Guidance Navigation, and Control Conference, AIAA 2005-5933, August 2005.

3. Belcastro, Christine M. and Foster, John V., "Aircraft Loss-of-Control Accident Analysis," AIAA Guidance Navigation, and Control Conference, AIAA 2010-8004, August 2010.

4. Advani, Sunjoo and Field, Joris, "Upset Prevention and Recovery Training in Flight Simulators," AIAA Modeling and Simulation Technologies Conference, AIAA 2011-6698, August 2011.

5. Morelli, Eugene A., "Efficient Global Aerodynamic Modeling from Flight Data," 50 ${ }^{\text {th }}$ Aerospace Sciences Meeting, AIAA 2012-1050, January 2012.

6. Murphy, P.C. and Klein, V., "Transport Aircraft System Identification Using Roll and Yaw Oscillatory Wind-tunnel Data," AIAA Atmospheric Flight Mechanics Conference, AIAA 2010-8122, August 2010.

7. Murphy, Patrick C. and Klein, Vladislav, "Progressive Aerodynamic Model Identification from Dynamic Water Tunnel Test of the F-16XL Aircraft," AIAA Atmospheric Flight Mechanics Conference, AIAA 2004-5277, August 2004.

8. Klein, V. and Murphy, P.C., "Estimation of Aircraft Nonlinear Unsteady Parameters from Wind Tunnel Data," NASA TM-1998-208969, December 1998.

9. Murphy, P.C. and Klein, V., "Estimation of Aircraft Unsteady Aerodynamic Parameters from Dynamic Wind Tunnel Testing," AIAA Atmospheric Flight Mechanics Conference, AIAA 2001-4016, August 2001.

10. Murphy, P.C. and Klein, V., "Validation of Methodology for Estimating Aircraft Unsteady Aerodynamic Parameters from Dynamic Wind Tunnel Tests," AIAA Atmospheric Flight Mechanics Conference, AIAA 2003-5397, August 2003.

11. Murphy, P.C., Klein, V., Frink, Neal T., and Vicroy, Dan D., "System Identification Applied to Dynamic CFD Simulation and Wind Tunnel Data," AIAA Atmospheric Flight Mechanics Conference, AIAA 2011-6522, August 2011. 
12. Vicroy, D. and Loeser, T., "SACCON Dynamic Wind Tunnel Tests at DNW- NWB and $14 \mathrm{ft} x 22 \mathrm{ft}$ NASA LaRC", AIAA Applied Aerodynamics Conference, AIAA-2010-4394, June 2010.

13. Frink, N. T., "Tetrahedral Unstructured Navier-Stokes Method for Turbulent Flows," AIAA Journal, Vol. 36, No. 11, November 1998, pp. 1975-1982.

14. Frink, N. T., Pirzadeh, S. Z., Parikh, P. C., Pandya, M. J.(2000) "The NASA Tetrahedral Unstructured Software System”. Aeronautical Journal, Vol. 104, No. 1040 (491-499). TetrUSS website: http://tetruss.larc.nasa.gov

15. Thompson, J.R., Frink, N.T., and Murphy, P. C., "Guidelines for Computing Longitudinal Dynamic Characteristics of a Subsonic Transport", AIAA Applied Aerodynamics Conference, AIAA 2010-4819, June 2010.

16. Tobak, M., and Schiff, L., "On the Formulation of the Aerodynamic Characteristics in Aircraft Dynamics," NASA TR R-456, 1976.

17. Klein, Vladislav and Norderer, Keith D., "Modeling of Aircraft Unsteady Aerodynamic Characteristics. Part I Postulated Models," NASA TM 109120, May 1994.

18. Klein, Vladislav, "Modeling of Longitudinal Unsteady Aerodynamics of a Wing-Tail Combination," NASA CR-1999209547, September 1999.

19. Klein, Vladislav, Murphy, P.C., and Szyba, Nathan M., "Analysis of Wind Tunnel Lateral Oscillatory Data of the F16XL Aircraft," NASA/TM-2004-213246, 2004.

20. Murphy, Patrick C. and Klein, Vladislav, "Estimation of Unsteady Aerodynamic Models from Dynamic Wind Tunnel Data," NATO, RTO-MP-AVT-189, 2011.

21. Klein, Vladislav and Morelli, Eugene, "Aircraft System Identification: Theory and Practice," AIAA Inc., Reston, Virginia, 2006.

\section{Figures}

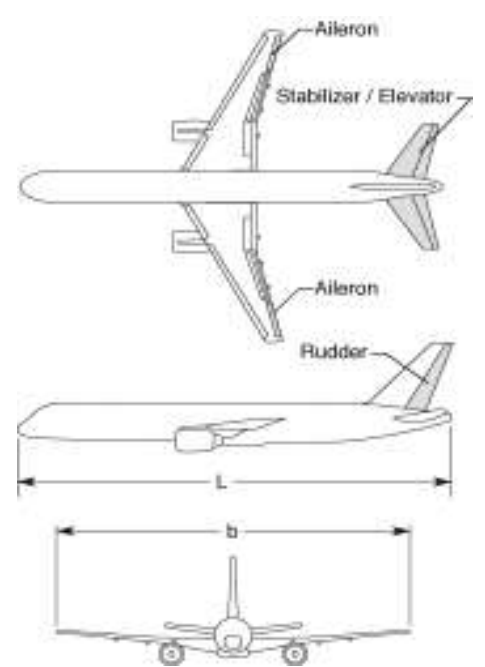

$\mathrm{S}=5.90 \mathrm{ft}^{2}, \bar{c}=0.915 \mathrm{ft}, \mathrm{b}=6.85 \mathrm{ft}$

Figure 1. Model geometry for NASA experimental subscale aircraft.

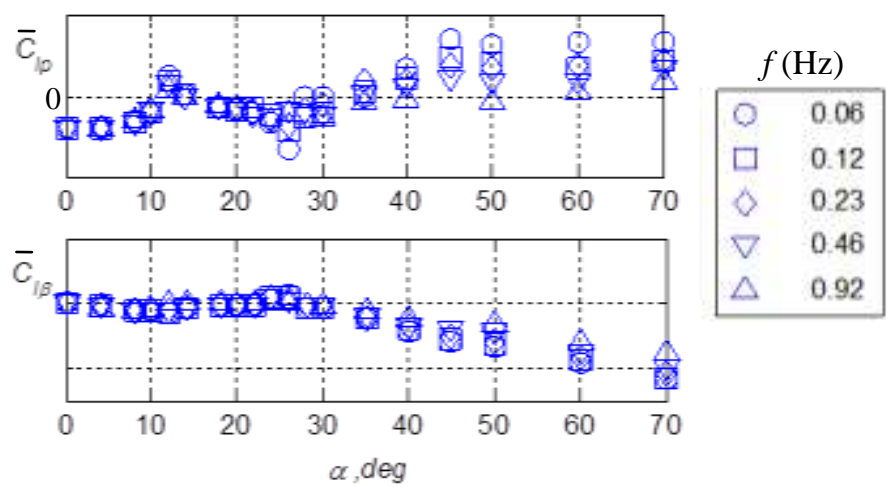

Figure 2a. Harmonic analysis for rolling moment coefficient, roll oscillations, $\phi_{A}=10^{\circ}$. 


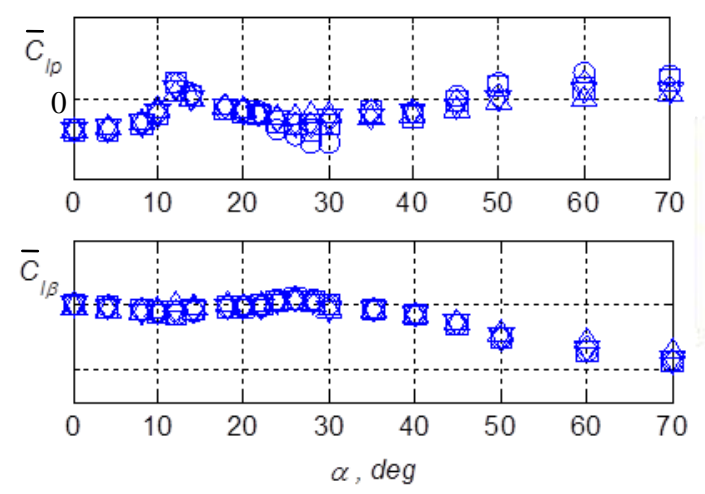

Figure 2b. Harmonic analysis for rolling moment coefficient, roll oscillations, $\phi_{A}=20^{\circ}$.

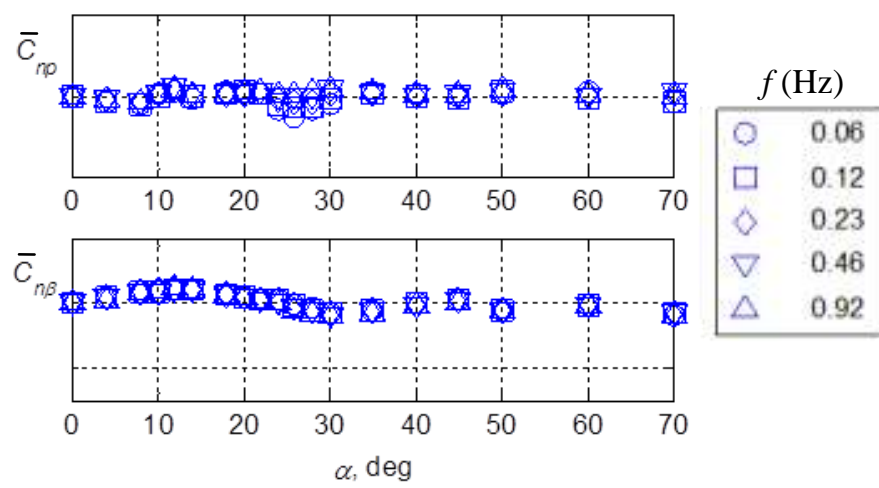

Figure 3a. Harmonic analysis for yawing moment coefficient, roll oscillations, $\phi_{A}=20^{\circ}$.
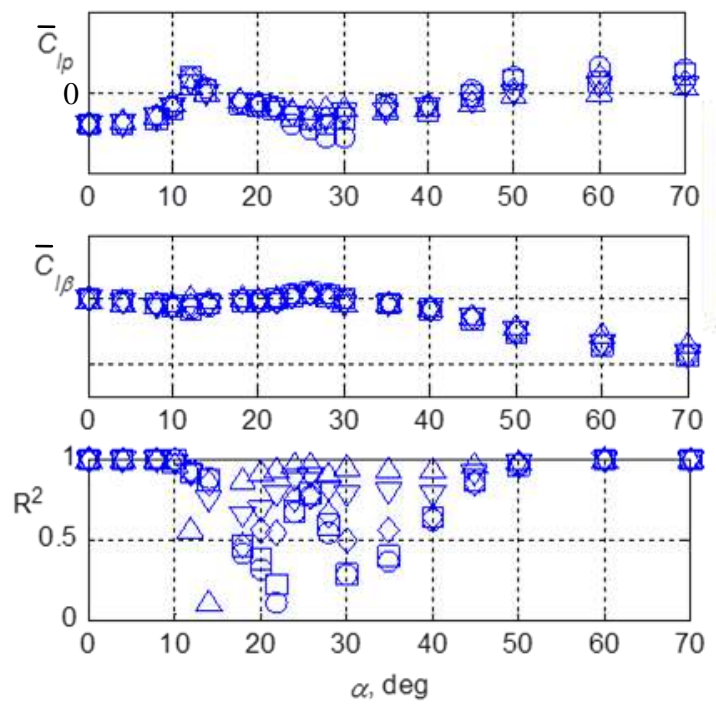

Figure 4a. Harmonic analysis for rolling moment coefficient, roll oscillations, $\phi_{A}=20^{\circ}(4 \mathrm{~Hz}$ filter).
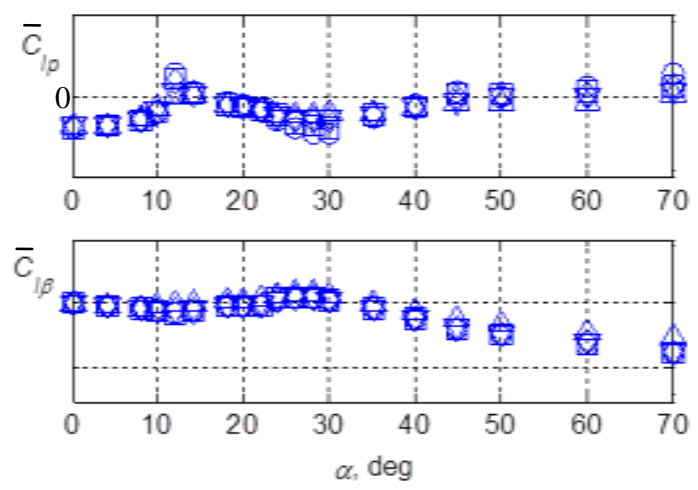

Figure 2c. Harmonic analysis for rolling moment coefficient, roll oscillations, $\phi_{A}=30^{\circ}$.

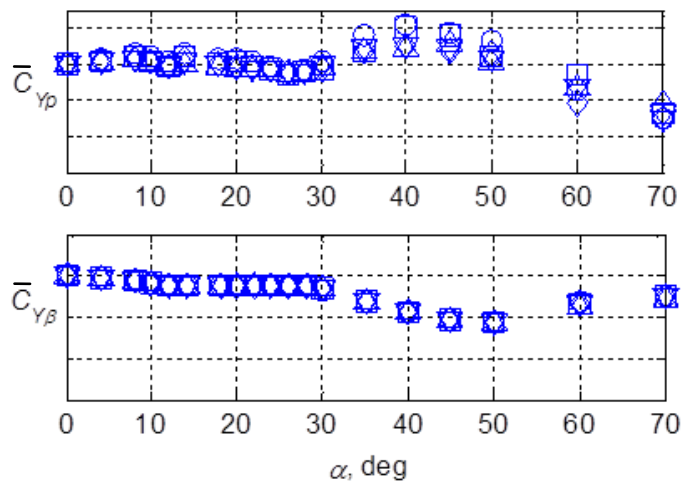

Figure 3b. Harmonic analysis for side force coefficient, roll oscillations, $\phi_{A}=20^{\circ}$.

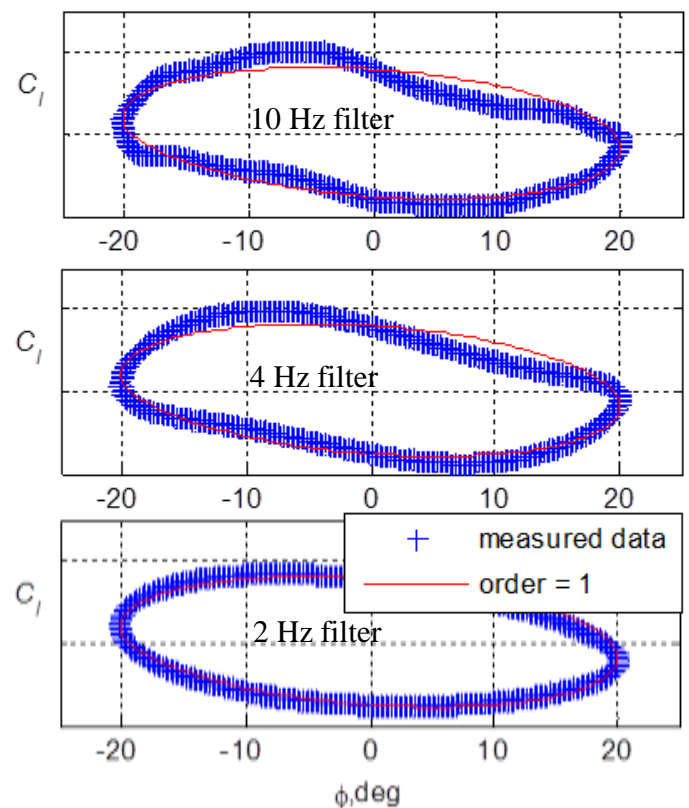

Figure 4b. Mean-cycle and first order harmonic model responses for rolling moment coefficient, roll oscillations, $\alpha_{0}=30^{\circ}, \phi_{A}=20^{\circ}$. 

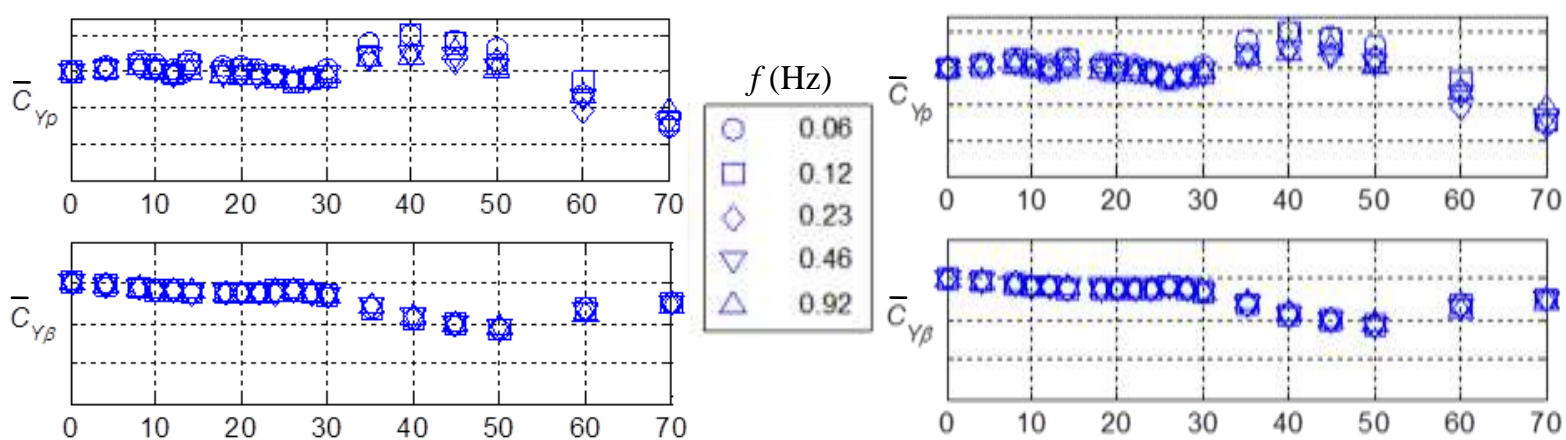

0.46

0.92
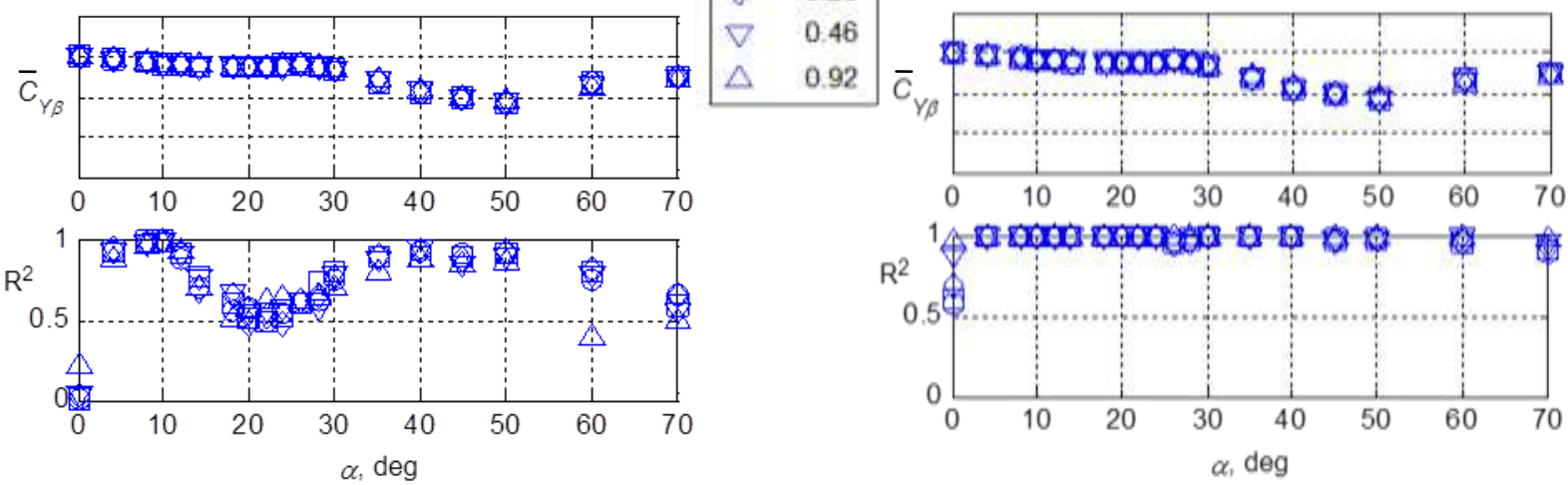

Figure 5a. Harmonic analysis for side force coefficient, roll oscillations, $\phi_{A}=20^{\circ}(10 \mathrm{~Hz}$ filter $)$.

Figure 5b. Harmonic analysis for side force coefficient, roll oscillations, $\phi_{A}=20^{\circ}$ ( $2 \mathrm{~Hz}$ filter $)$.
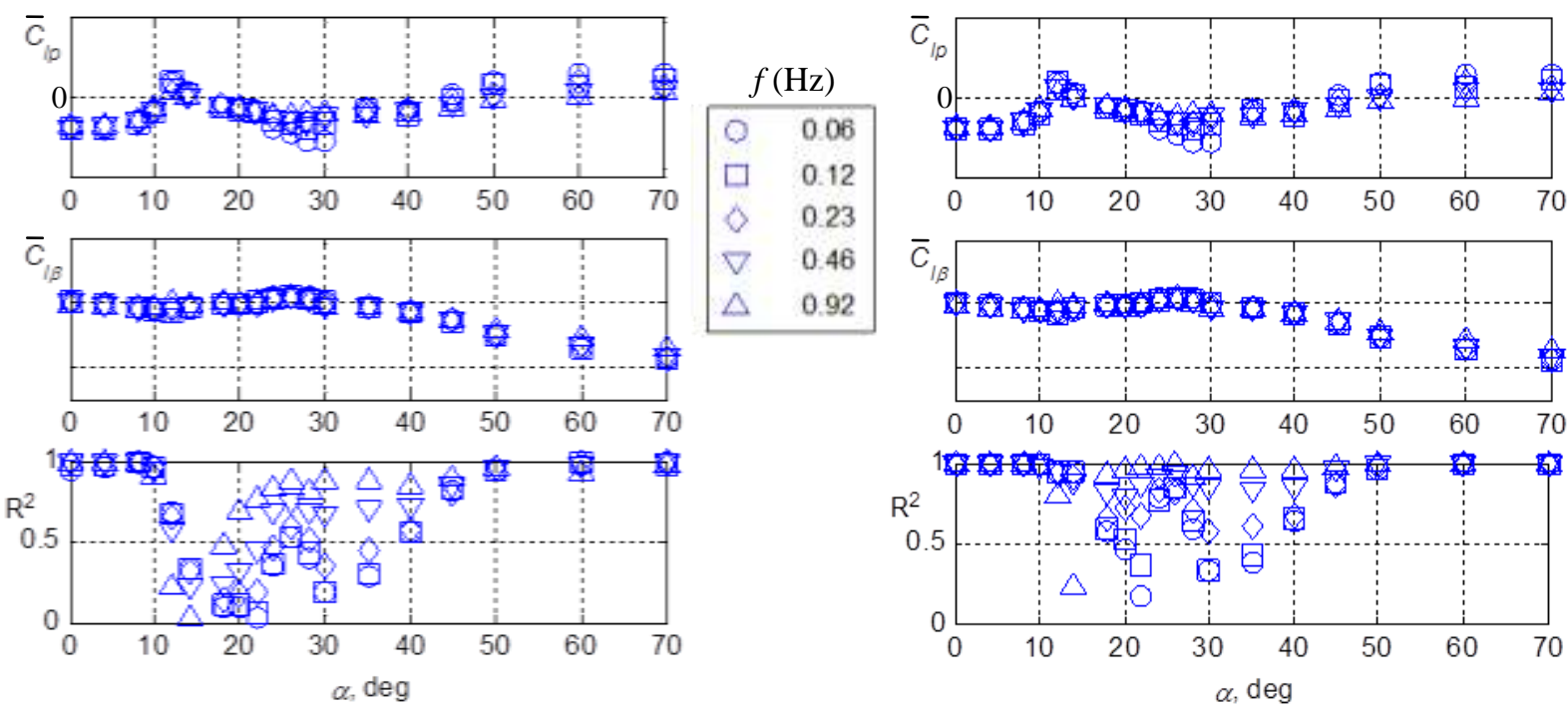

Figure 6a. Harmonic analysis for rolling moment

Figure 6b. Harmonic analysis for rolling moment coefficient, roll oscillations, $\phi_{A}=20^{\circ}(10 \mathrm{~Hz}$ filter $)$. coefficient, roll oscillations, $\phi_{A}=20^{\circ}(2 \mathrm{~Hz}$ filter $)$. 

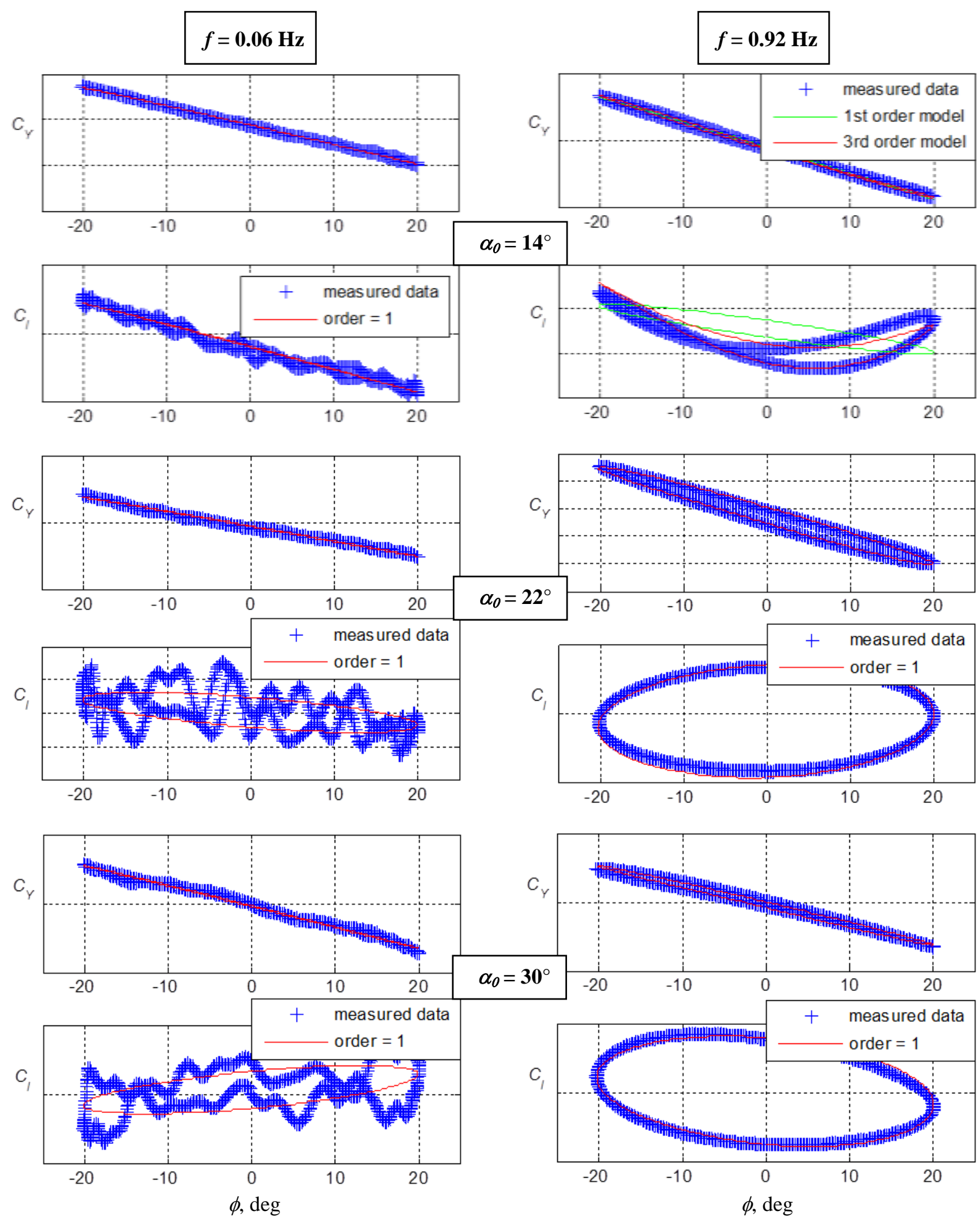

Figure 7. Mean cycle and first order harmonic model responses, with amplitude $\phi_{A}=20^{\circ}(2 \mathrm{~Hz}$ filter). 
$\mathbf{f}=0.92 \mathrm{~Hz}$
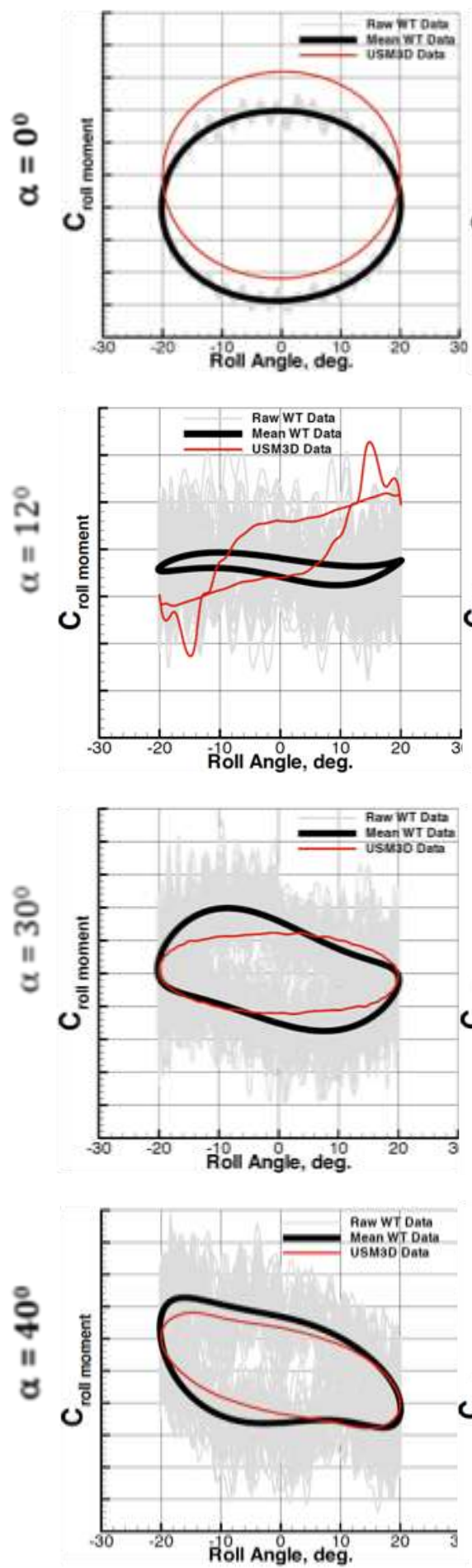

$\mathbf{f}=0.12 \mathrm{~Hz}$
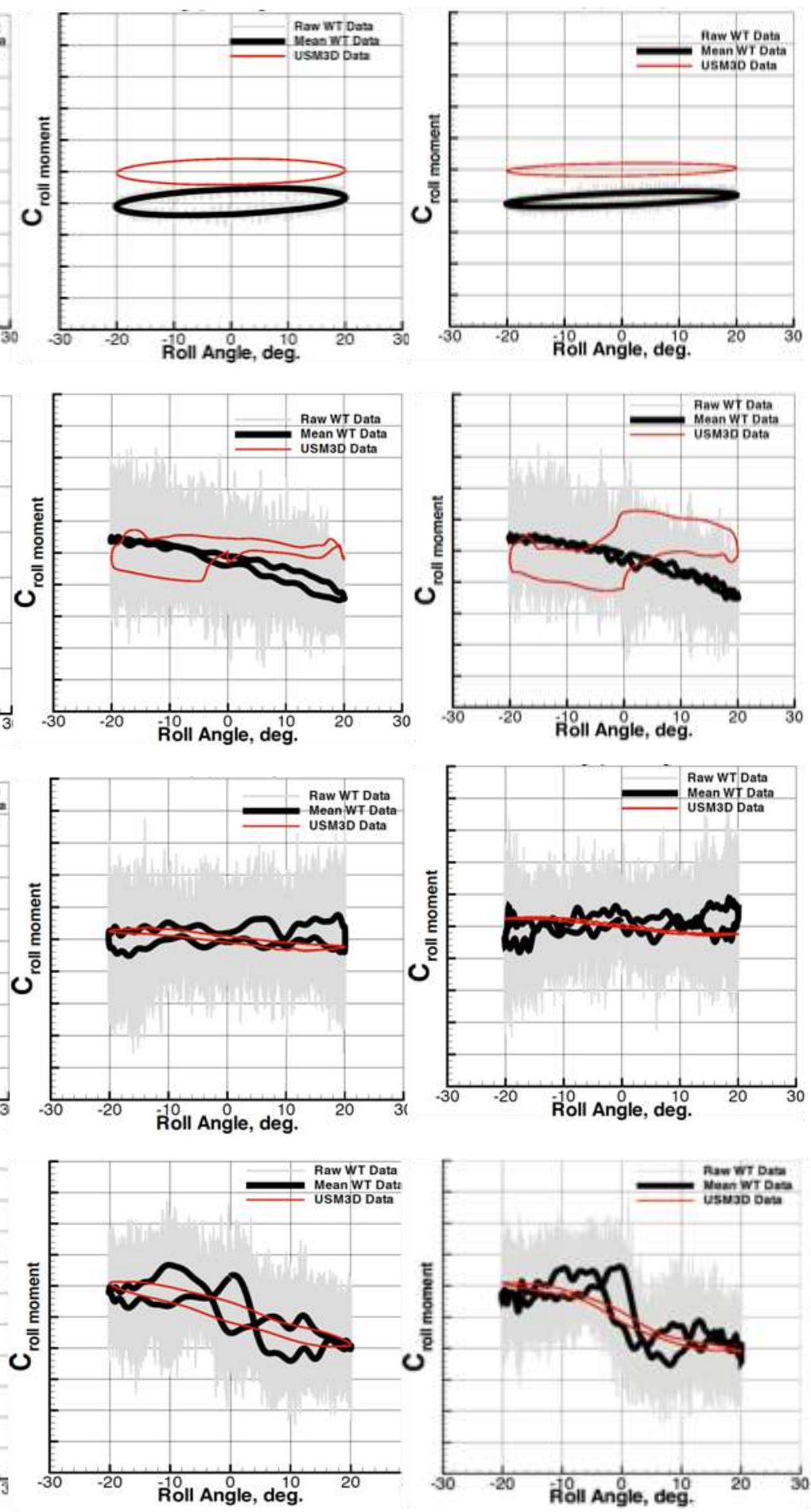

$\mathbf{f}=\mathbf{0 . 0 6 ~ H z}$
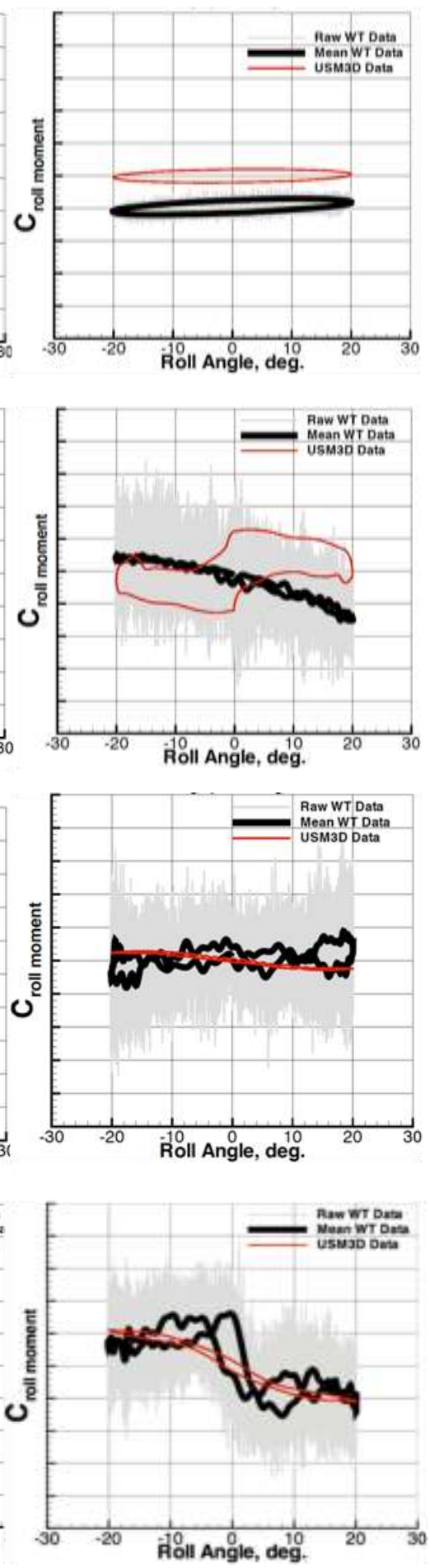

Figure 8. Rolling moment forced oscillations from CFD and wind tunnel (unfiltered and mean-cycle) data, with amplitude $\phi_{A}=20^{\circ}$, for GTM over range of angle of attack and frequency. 


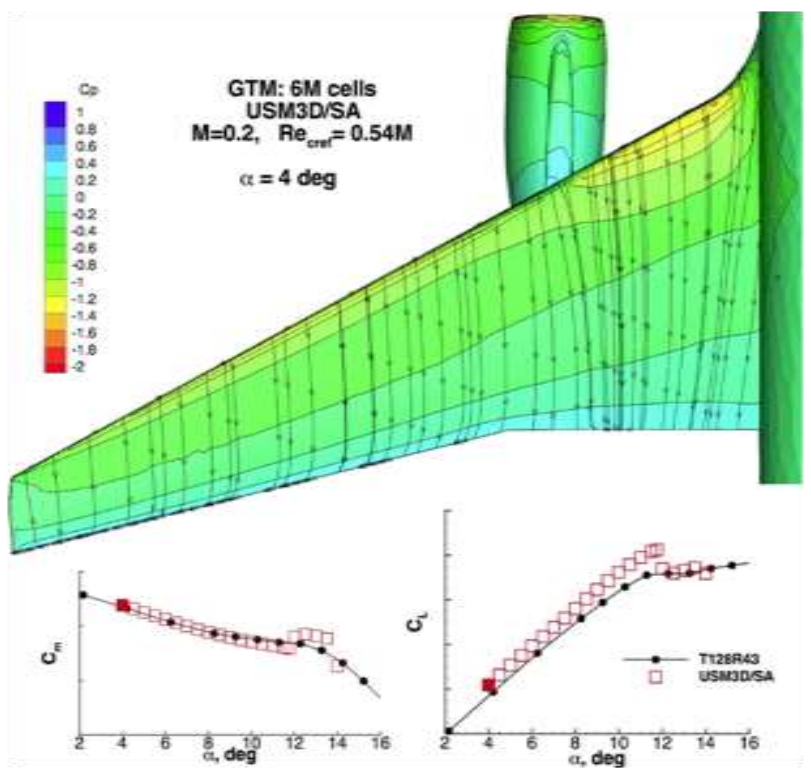

Figure 9a. CFD calculation of streamlines for GTM at $\alpha=4^{\circ}$.

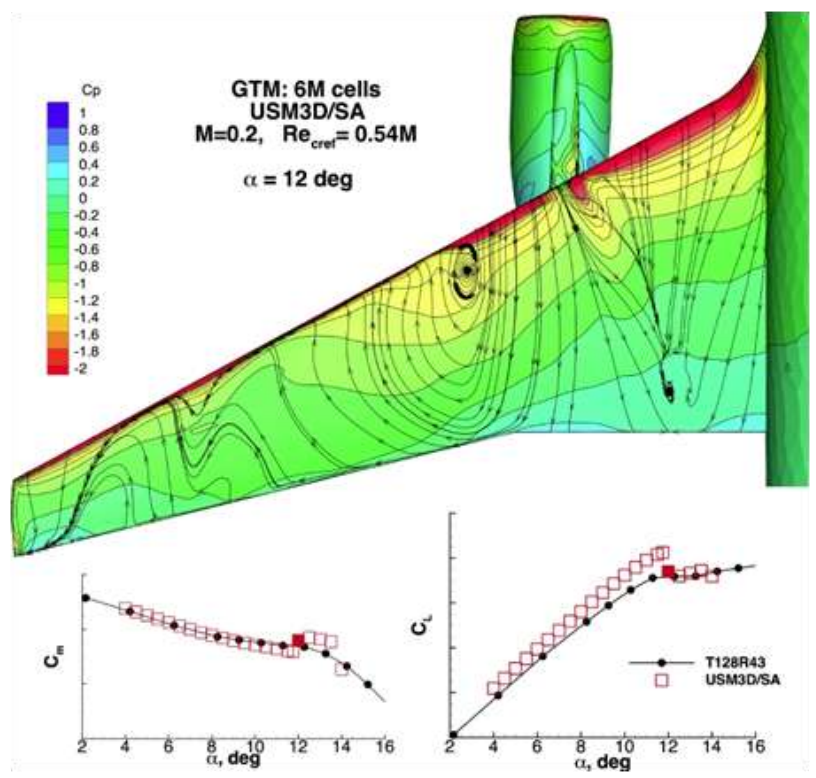

Figure 9c. CFD calculation of streamlines for GTM at $\alpha=12^{\circ}$.

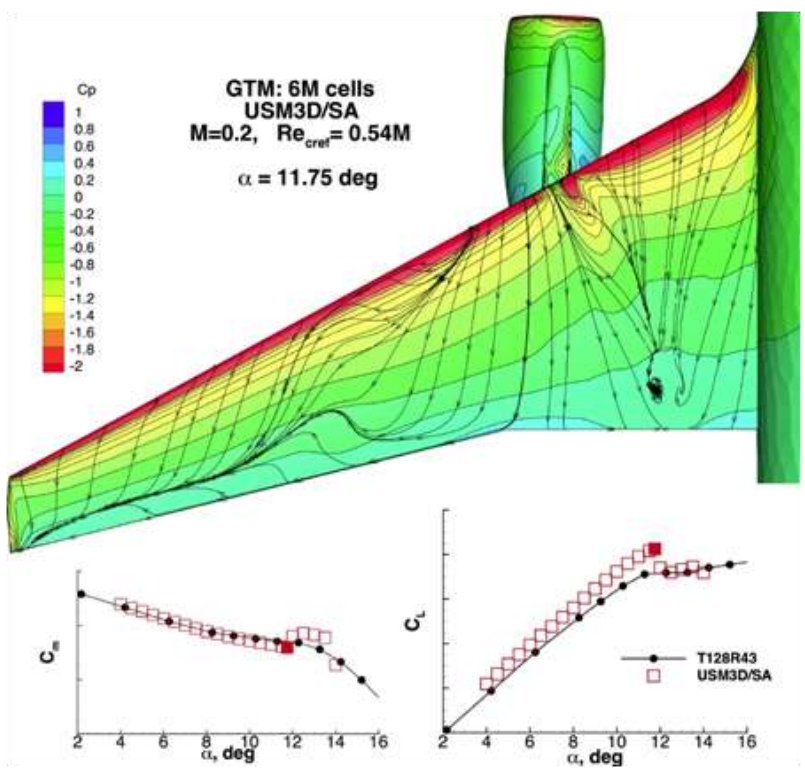

Figure 9b. CFD calculation of streamlines for GTM at $\alpha=11.75^{\circ}$.

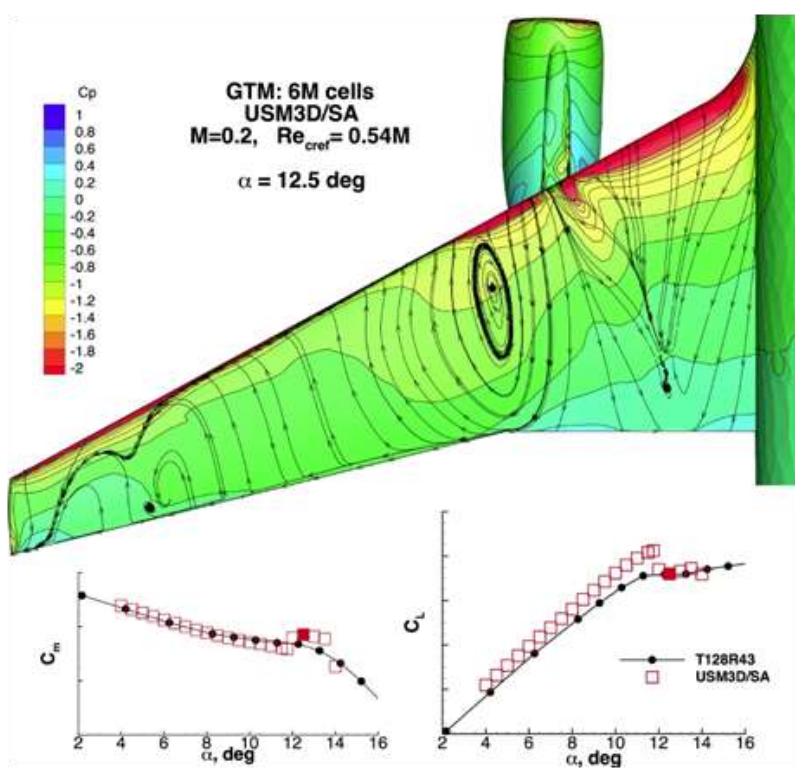

Figure 9d. CFD calculation of streamlines for GTM at $\alpha=12.5^{\circ}$. 

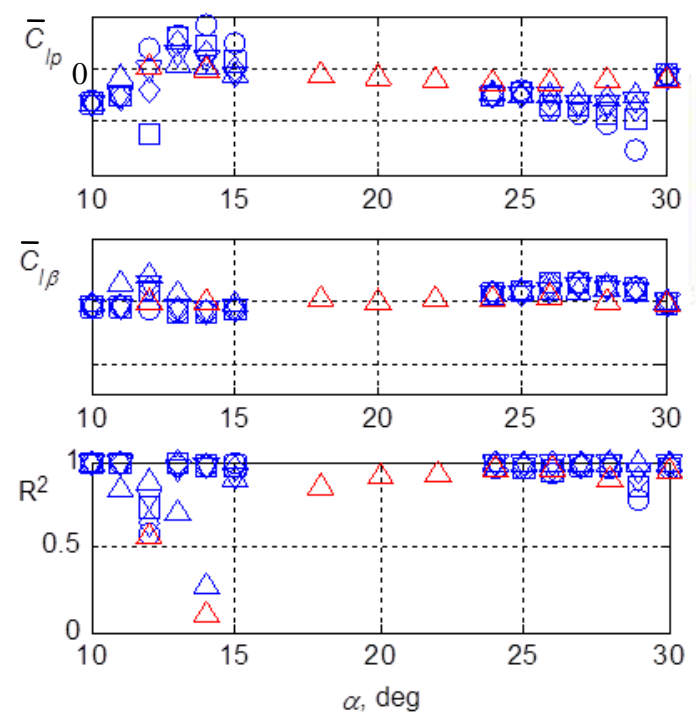

Figure 10. Harmonic analysis for CFD simulated rolling moment coefficient, roll oscillations, $\phi_{A}=20^{\circ}$ (4 Hz filter applied to wind tunnel data).

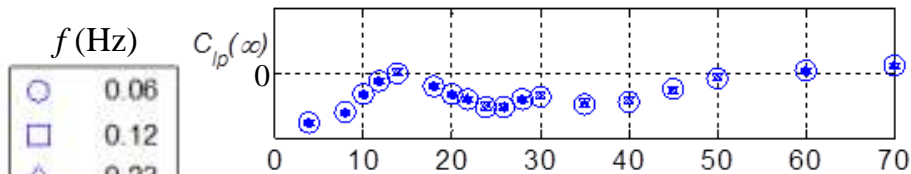

a

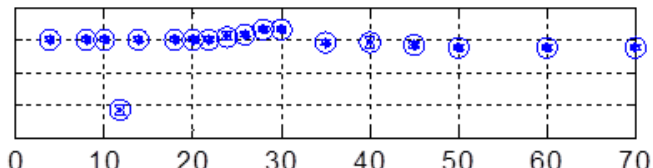

Wind tunnel data at $0.92 \mathrm{~Hz}$

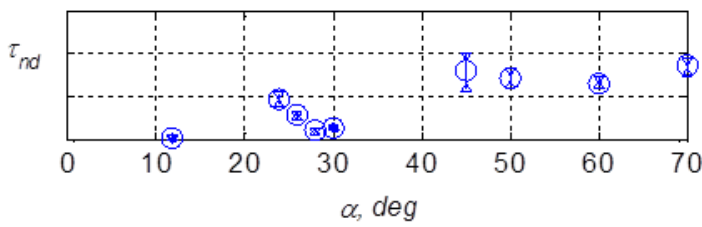

Figure 11. Unsteady model parameters with $2 \sigma$ uncertainty bounds for rolling moment coefficient, $\phi_{A}=20^{\circ}$.
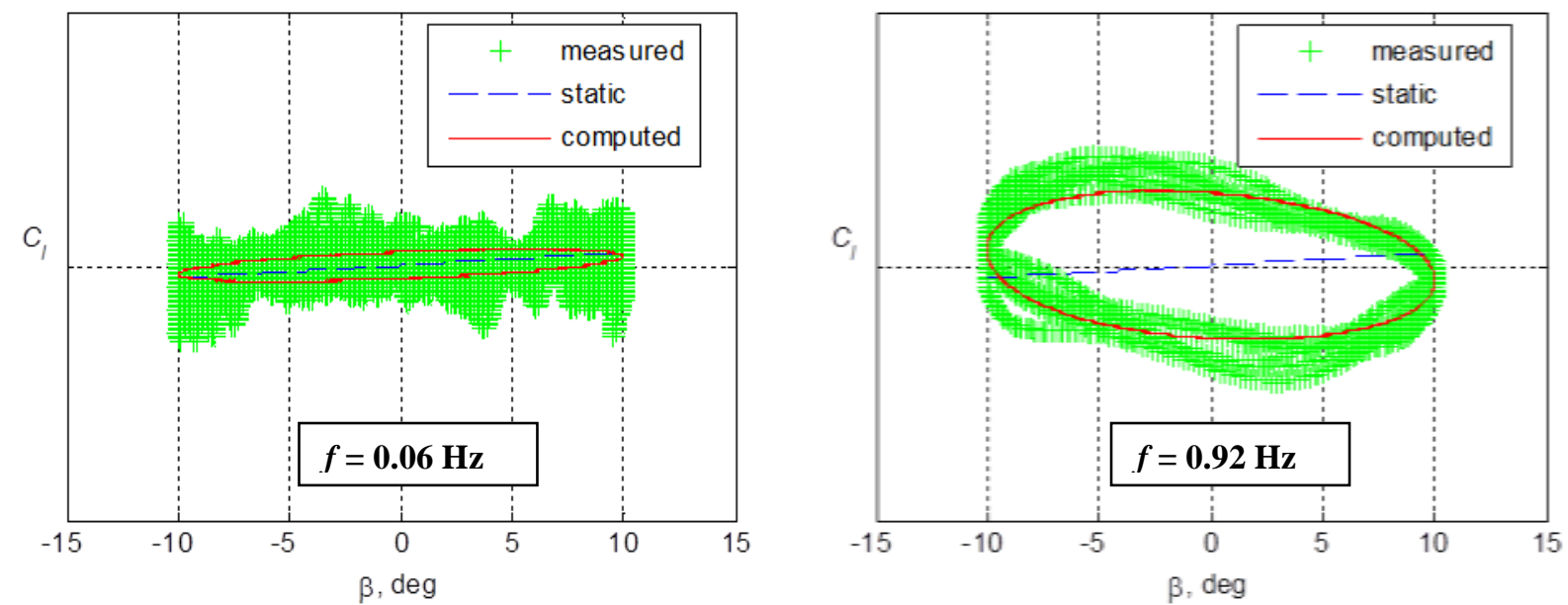

Figure 12. Unfiltered measurements and unsteady model responses for rolling moment coefficient, at two oscillation frequencies, $\alpha=30^{\circ}, \phi_{A}=20^{\circ}$. 